\title{
Diagnostic Role of Serum Neutrophilic/Lymphocytic and Platelet/Lymphocytic Ratio in upper Gastrointestinal Lesions in Correlation with Histopathological Results of Endoscopic Ultrasonography and Upper Gastrointestinal Endoscopic Biopsies
}

\author{
ESSAM M. FARID, M.D.; WAEL A. YOUSRY, M.D.; SHEREEN A. SALEH, M.D. and \\ SARAH S. SALEM ASAR, M.Sc.
}

The Department of Gastroenterology \& Internal Medicine, Faculty of Medicine, Ain Shams University, Cairo, Egypt

\begin{abstract}
Background: Systemic inflammatory markers including neutrophilic lymphocytic ratio and platelet lymphocytic ratio are used as prognostic and diagnostic factors to reflect the inflammatory process and tumor angiogenesis in many tumors including upper gastrointestinal submucosa tumors.
\end{abstract}

Aim of Study: To determine the diagnostic efficacy of the Neutrophil-to-Lymphocyte Ratio (NLR) and Platelet-toLymphocyte Ratio (PLR) in patients with uppergastrointestinal submucosal tumors.

Patients and Methods: The present study included 30 patients with malignant and potentially malignant upper gastrointestinal submucosal tumor, 30 with benign upper gastrointestinal submucosal tumor and 30 healthy volunteers, complete blood counts with automated differential counts were performed. The NLR was calculated by dividing the absolute neutrophilic count by the absolute lymphocytic count while PLR was calculated by dividing the absolute platelet count by the absolute lymphocytic count. The diagnostic performance of NLR, PLR, was estimated by ROC curve.

Results: ROC curve analysis showed high diagnostic efficacy of NLR and PLR in malignant and potentially malignant submucosal tumors patients with cut-off values of 2.25 ( sensitivity $=60 \%$, and specificity $=86.7$, PPV $81.9 \%$, NPV $68.4 \%$ ) and 169 (sensitivity $=93.3 \%$, and specificity $=100 \%$ PPV 100\%, NPV 93.7\%) compared to patients with benign lesions and healthy controls, respectively.

Conclusion: NRL, PLR, may be useful markers in diagnostic and early recognition of different upper gastrointestinal submucosal lesions.

Key Words: Submucosal tumors - Neutrophilic lymphocytic ratio-Platelet lymphocytic ratio-Diagnosis.

Correspondence to: Dr. Sarah S. Salem Asar, The Department of Gastroenterology \& Internal Medicine, Faculty of Medicine, Ain Shams University, Cairo, Egypt

\section{Introduction}

DESPITE advances in the diagnostic technology, Subepithelial Lesions (SELs) in the upper Gastrointestinal (GI) remain a diagnostic challenge for the endoscopist as they lay below the epithelial layer.

The majority of subepithelial lesions are benign at though many of these neoplasms have potential for malignant transformation and spread to distant organs [1]

Endoscopic ultrasonography is the primary established diagnostic tool for submucosal lesions. Though in recent years, there has been an interest in the use of inflammatory markers for the early detection of malignancies.

Inflammation can initiate the onset of cancer, its progression and metastasis. Neutrophils, platelets, and T-and B-lymphocytes all are considered the backbone of tumor related inflammation and immunology. A number of inflammatory markers have been studied in different cancer types to determine their early diagnostic efficacy and prediction of prognosis. Including the Neutrophil/ Lymphocyte Ratio (NLR), the Platelet/Lymphocyte Ratio (PLR), and the Mean Platelet Volume (MPV). PLR, NLR, and MPV have been reported to be increased in patients with a number of cancer types, including. Colorectal cancer, gastric cancer and other organ solid malignancies as ovarian cancer [2]

\section{Patients and Methods}

During the period between February 2018 and January 2019 , a cross sectional study conducted 
in National Hepatology and Tropical Medicine Research Institute, 90 individuals are included and divided into three groups:

- Thirty patients who were diagnosed with upper gastrointestinal tract malignant or potentially malignant submucosal/sub epithelial tumors.

- Thirty patients who were diagnosed with upper gastrointestinal tract benign submucosal/ subepithelial tumors.

- Thirty subjects referred to as the control group collected from the out-patient clinic matching the patients in age and sex representing the healthy control.

\section{Inclusion criteria:}

- Patients complaining of dyspeptic and epigastric pain, suspecting upper gastrointestinal tract submucosal lesions after performing conventional upper endoscopy with non-significance mucosal biopsy and were sent for EUS examination.

All patients gave their informed verbal consent and the ethics committee of our unit approved the study protocol.

\section{Exclusion criteria:}

- Patient under age of 18.

- Chronic cardiac, lung, renal or liver diseases.

- Patients treated with steroids or immunosuppressant for the last year.

- Pancreatic, hepatic, adrenal, and mediastinal lesions, as well as peri-GI tract lymphadenopathies, were excluded from the study.

\section{All included patients were subjected to the following: \\ - Full clinical history and physical examination with special emphasis on manifestation of upper GIT symptoms including epigastric pain, loss of appetite, vomiting, bowel habit change, hemate- mesis or melena together with epigastric masses or tenderness.}

\section{- Laboratory investigations:}

- Complete Blood Counts (CBC) with automated differential counts were performed where peripheral blood $(2 \mathrm{~mL})$ samples were collected from the cubital vein. CBC analysis was performed in samples anticoagulated with EDTA within 4 hours after collection, using Mindray BC-3200 Hematology Analyzer.

- The NLR was calculated by dividing the Absolute Neutrophil Count (ANC) by the Absolute Lymphocyte Count (ALC); likewise, PLR was calculated by dividing the absolute platelet count by ALC.

- Tumor markers including: CEA (carcinoembryonic antigen) and CA19-9 (carboni anhydrase 19-9).

- Computed Tomography (CT scan) with contrast \pm Dynamic MRI (Magnetic Resonance Imaging: To assess in diagnosis with EUS.

\section{- Upper gastrointestinal endoscopy and mucosal} biopsies:

- All the patients underwent upper gastrointestinal tract endoscopy, after 8-10-hour fast, using Olympus Evis lucera 260 and forceps biopsies were taken by the same endoscopist with no diagnostic significance and they were referred for EUS examination.

- EUS examination \pm EUS guided (FNA) fine needle aspiration.

For all patients, after an overnight fast and under light sedation after 8-10-hour fast, every patient received $5 \mathrm{mg}$ of midazolam \pm local pharyngeal anesthesia (xylocaine spray) was done \pm propofol was used for irritable patients under supervision of anaesthiologist with the patient lying on left lateral position EUS was performed.

\section{- The echoendoscope:}

A linear scanning echoendoscope, Pentax EG 3830UT/ HITACHI 7500. The transducer is mounted at the tip of the echoscope. Its acoustic frequency is switchable from 5 to $12.5 \mathrm{MHz}$. The scan angle is 270 degree.

\section{- The display unit:}

It consists of Hitachi EUB 7500 ultrasound scanner system with a built in color flow mapping (CAM) and Doppler function.

\section{- The technique of examination $\pm F N A C$ :}

Patients were fasting (an overnight fast) before the examination.

The endoscope was connected to the ultrasound display unit after being cleaned and sterilized as with other ordinary endoscopes and introduced, guided through the stomach and down to the second part of the duodenum to evaluate and stage GI lesions.

Once a lesion was identified, EUS guided FNA using a 22-gauge needle was performed if needed by moving the needle back and forth within the target lesion for 15-30 seconds. No suction was applied during biopsy unless the biopsy did not 
yield any material or the lesion was cystic after Color flow and Doppler sonography to exclude intervening vascular structures and select a vesselfree needle track.

The aspirate was placed on glass slides, and both air-dried and alcohol-fixed smears were prepared. Air dried smears were stained with a modified Giemsa stain and reviewed by a cytopathologist.

- Cytological examination: The original cytologic diagnoses were categorized as follows:

Positive for a neoplasm or suspicious for a neoplasm and non-neoplastic/reactive/ inflammatory process according to cytologic preparations as smear pattern, palisading, crush artifact, prominent vascular pattern, nuclear grooves/inclusions, pleomorphism, morphology (spindle, epithelioid, round cels, etc..), blunt-ended or wavy nuclei, perinuclear vacuoles, cytoplasmic quality, mitoses, and necrosis, atypia or dysplasia, in addition to mitosis in GIST for instance.

The results were confirmed by histologic and pathological examination of the surgically removed and endoscopically resected pathologic specimens.

To determine the NLR, PLR in diagnosing GI tract neoplasms, sensitivity, specificity, and diagnostic accuracy were calculated compared to the histological and pathological yield of the EUS imaging \pm EUS guided FNA results.

\section{- Statistical analysis:}

Data were coded and entered using the SPSS (Statistical Package for the Social Sciences; SPSS Inc., Chicago, Illinois, USA) Version 24. Data were summarized using mean, $\mathrm{SD}$, median, minimum, and maximum in quantitative data and using frequency and percentage for categorical data. A $p$-value of less than 0.05 indicated statistical significance. Differences between the groups were eyaluated by an independent sample $t$-test and a $\chi$-test. Receiver operating characteristic curve was constructed with area under curve analysis performed to detect the best cutoff value of NLR and PLR for diagnosis gastrointestinal submucosal tumors.

\section{Results}

The current study included 30 patients with malignant and potentially malignant submucosal tumor, 30 patient with benign submucosal tumor and 30 healthy control.
In the malignant/potentially malignant group it was found that gastrointestinal stromal represented the majority of cases followed by adenocarcinoma, lymphoma and leiyomyosarcoma.

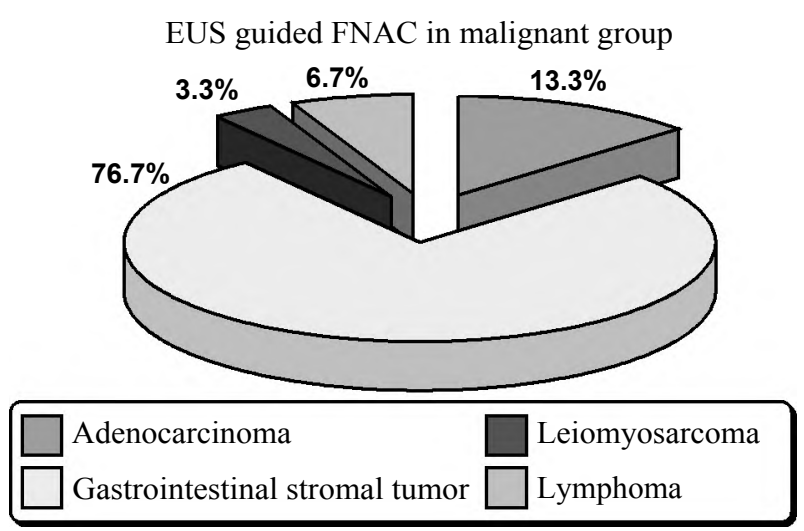

Fig. (1): Description of EUS guided FNAC in malignant group.

$$
\text { EUS, } \pm \text { EUS guided FNAC in benign group }
$$

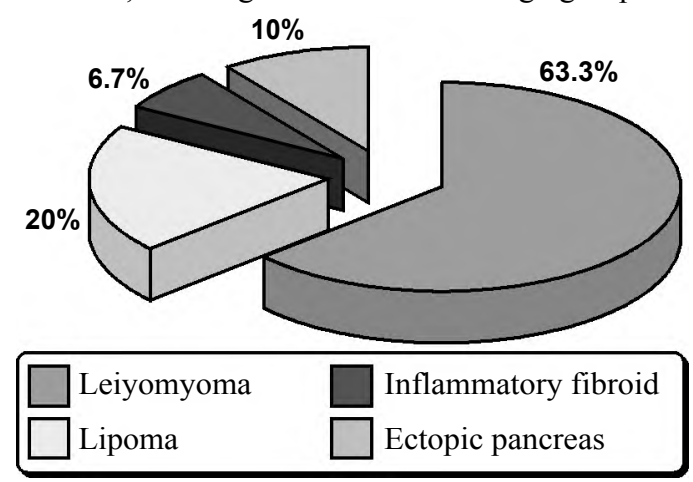

Fig. (2): Description of EUS \pm FNAC in benign group.

While for the benign group, the majority of the cases was found to be leiyomyoma, lipoma, inflammatory fibroid and ectopic pancreas.

Table (1): Comparison between studied groups as regard lymphocyte count.

\begin{tabular}{lrrrr}
\hline Variables & $\begin{array}{c}\text { Malignant/ } \\
\text { potentially } \\
\text { malignant }(\mathrm{N}=30)\end{array}$ & $\begin{array}{c}\text { Benign } \\
(\mathrm{N}=30)\end{array}$ & $\begin{array}{c}\text { Control } \\
(\mathrm{N}=30)\end{array}$ & $p$-value \\
\hline ALC (cell/ul): & & & & \\
Mean $\pm \mathrm{SD}$ & $1990.93 \pm 1046.16$ & 2195.4 & 2505.6 & $p 0=0.1$ \\
& & \pm 702.1 & \pm 1013.6 & $p 1=0.4$ \\
& & & $p 2=0.04 *$ \\
& & & $p 3=0.2$ \\
\hline
\end{tabular}

It was found that absolute lymphocytic count has a statistical difference between the malignant/ potentially malignant group and the benign group being the lower at the first group of patient and between the malignant value of all in the first malignant/potentially malignant group and the healthy control group being higher at the latter too while there is no statistical difference between the 
benign group and the healthy control regarding ALC.

Table (2): Comparison between studied groups as regard absolute neutrophil count.

\begin{tabular}{|c|c|c|c|c|}
\hline Variables & $\begin{array}{c}\text { Malignant/ } \\
\text { potentially } \\
\text { malignant }(\mathrm{N}=30)\end{array}$ & $\begin{array}{l}\text { Benign } \\
(\mathrm{N}=30)\end{array}$ & $\begin{array}{l}\text { Control } \\
(\mathrm{N}=30)\end{array}$ & $p$-value \\
\hline \multicolumn{5}{|l|}{ ANC (cell/ul): } \\
\hline & $4941.4 \pm 2404.2$ & $\begin{array}{c}3704.6 \pm \\
1716.5\end{array}$ & $\begin{array}{l}3391.9 \pm \\
1086.5\end{array}$ & $\begin{array}{l}p 0=0.003 * \\
p 1=0.01 * \\
p 2=0.001 * \\
p 3=0.5\end{array}$ \\
\hline
\end{tabular}

The absolute neutrophilic count was found to be significantly higher in the malignant/potentially malignant group than that of the benign group as well as healthy controls, while there is no statistical difference between the benign group and the healthy control. There was no significant difference between the three groups regarding the total leuckocytic count.

Table (3): Comparison between studied groups as regard PLT count.

\begin{tabular}{lllll}
\hline Variables & $\begin{array}{c}\text { Malignant/ } \\
\text { potentially } \\
\text { malignant }(\mathrm{N}=30)\end{array}$ & $\begin{array}{c}\text { Benign } \\
(\mathrm{N}=30)\end{array}$ & $\begin{array}{l}\text { Control } \\
(\mathrm{N}=30)\end{array}$ & $p$-value \\
\hline$P L T\left(X 10^{3} / U L\right):$ & & & & \\
Mean $\pm \mathrm{SD}$ & $348.87 \pm 371.44$ & 194.20 & 224.96 & $p 0=0.02$ \\
& & \pm 75.83 & \pm 79.74 & $p 1=0.009$ \\
& & & & $p 2=0.04 *$ \\
& & & & $p 3=0.6$ \\
\hline
\end{tabular}

Platelet count was found to be significantly higher in the first malignant/potentially malignant group than that of the benign group and the healthy controls while there is no statistical difference in platelet count between healthy controls and the benign group.

Table (4): Comparison between studied groups as regard NLR.

\begin{tabular}{lcccc}
\hline Variables & $\begin{array}{c}\text { Malignant/ } \\
\text { potentially } \\
\text { malignant }(\mathrm{N}=30)\end{array}$ & $\begin{array}{c}\text { Benign } \\
(\mathrm{N}=30)\end{array}$ & $\begin{array}{c}\text { Control } \\
(\mathrm{N}=30)\end{array}$ & $p$-value \\
\hline$N L R:$ & & & & \\
Mean $\pm \mathrm{SD}$ & $2.9 \pm 1.39$ & $1.8 \pm 0.5$ & $1.5 \pm 0.4$ & $\begin{array}{l}p 0<0.001 \\
p 1<0.001 * \\
p 2<0.001 \\
\end{array}$ \\
& & & & $\begin{array}{l}p=0.2 \\
\end{array}$ \\
\hline
\end{tabular}

As for neutrophilic lymphocytic ratio it was found to be significantly higher in the malignant and potentially malignant group with a mean value $2.9 \pm 1.39$ than the other two groups and there was no statistical difference between the benign and the healthy control groups.
Table (5): Comparison between studied groups as regard PLR.

\begin{tabular}{lcccc}
\hline Variables & $\begin{array}{c}\text { Malignant/ } \\
\text { potentially } \\
\text { malignant }(\mathrm{N}=30)\end{array}$ & $\begin{array}{c}\text { Benign } \\
(\mathrm{N}=30)\end{array}$ & $\begin{array}{c}\text { Control } \\
(\mathrm{N}=30)\end{array}$ & $p$-value \\
\hline$P L R:$ & & & & \\
Mean $\pm \mathrm{SD}$ & $221.9 \pm 40.8$ & 137.3 & $88.8 \pm$ & $p 0<0.001^{*}$ \\
& & \pm 18.5 & 41.84 & $p 1<0.001^{*}$ \\
& & & $p 2<0.001^{*}$ \\
& & & & $3<0.001^{*}$ \\
\hline
\end{tabular}

Platelet lymphocytic ratio showed a statistical difference between the malignant/potentially malignat group and the benign group as well as the healthy controls with being significantly higher at the first group with a mean value $221.9 \pm 40.8$ and between benign healthy control being higher at the first group too.

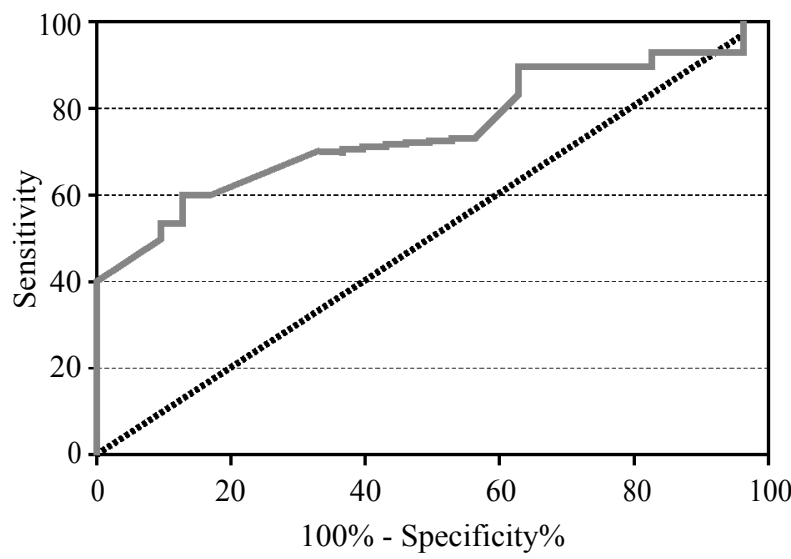

Fig. (3): ROC curve between malignant group and benign group as regard NLR.

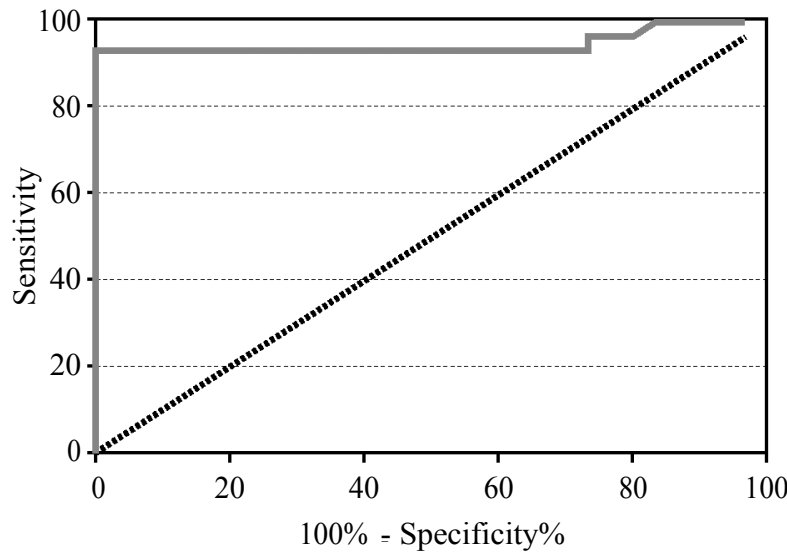

Fig. (4): ROC curve between malignant group and benign group as regard PLR.

Using roc curve, NLR can be used to discriminate between malignant group and benign group at a cutoff level of $>2.25$, with $60 \%$ sensitivity, $86.7 \%$ specificity, $81.9 \%$ PPV and $68.4 \%$ NPV.

Using roc curve, PLR can be used to discriminate between malignant group and benign group 
at a cutoff value $>169$, with $93.3 \%$ sensitivity, $100 \%$ specificity, $100 \% \mathrm{PPV}$ and $93.7 \% \mathrm{NPV}$.

Table (6): Diagnostic performance of NLR in discrimination of malignant group and benign group.

\begin{tabular}{lcccccc}
\hline $\begin{array}{l}\text { NLR } \\
\text { cut off }\end{array}$ & $\begin{array}{c}\text { Area under } \\
\text { the curve }\end{array}$ & Sensitivity & Specificity & PPV & NPV & $\begin{array}{c}p \text { - } \\
\text { value }\end{array}$ \\
\hline$>2.25$ & 0.7 & $60 \%$ & $86.7 \%$ & $81.9 \%$ & $68.4 \%$ & 0.0009 \\
\hline
\end{tabular}

Table (7): Diagnostic performance of PLR in discrimination of malignant group and benign group.

\begin{tabular}{lcccccc}
\hline $\begin{array}{l}\text { PLR } \\
\text { cut off }\end{array}$ & $\begin{array}{c}\text { Area under } \\
\text { the curve }\end{array}$ & Sensitivity & Specificity & PPV & NPV & $\begin{array}{c}p- \\
\text { value }\end{array}$ \\
\hline$>169$ & 0.9 & $93.3 \%$ & $100 \%$ & $100 \%$ & $93.7 \%$ & $<0.001$ \\
\hline
\end{tabular}

\section{Discussion}

Subepithelial Lesions (SELs) in the upper Gastrointestinal (GI) tract are relatively frequent findings in patients undergoing an upper GI endoscopy that posses a diagnostic challenge for the gastroenterologist as they lay below the epithelial layer. The majority of subepithelial lesions are benign at the time of diagnosis, with fewer than $15 \%$ of lesions found to be malignant at presentation [1]

Despite this, many of these neoplasms have potential for malignant transformation and spread to distant organs. The key in evaluating patients with subepithelial lesions is to identify the definitive diagnoses and the type of lesion together with the early detection of the malignancy in order to determine the proper therapies, prognosis and need for surveillance [3]

An endosonographic evaluation using EUS in addition to cytological finding by EUS guided FNAC allows the differentiation of extrinsic lesions from intramural lesions, in addition to identification of the originating layer, characterization of echo features, and tissue acquisition to reach an accurate diagnosis $[3,4]$.

The decision can be based on EUS features like the size, echogenicity and location in the GI tract, \pm the cytological yield of the FNAC where the presence of typical endosonographic benign features (e.g., vascular structures, cysts, and lipoma) denies the need for tissue acquisition, follow-up, and resection.

However, the lack of the extension, accessibility and feasibility of these screening and diagnostic methods in the developing countries remains an obstacle towards presuming early diagnosis and improving the survival rates [5].

Detection of GI malignancies rates are higher in some countries like Japan and Western countries than in developing countries yielding in higher survival rates [6].

This study was concerned with the role of systemic inflammation in the process of carcinogenesis, where few studies reported their relation with diagnosis and early detection of malignancies.

The inflammatory markers including the value of Absolute Neutrophilic Count (ANC), Absolute Lymphocytic Count (ALC), Neutrophil Lymphocytic Ratio (NLR) and Platelet Lymphocytic Ratio (PLR) were evaluated in malignant and potentially malignant submucosal lesions and compared with the benign submucosal ones and with normal individuals.

ALC was significantly lower in malignant group than in benign group as well as the control group while there is no significant difference between the latter two groups. This is can be explained by the action of the increased neutrophilic count specifically which inhibit the anti-tumor immune response by suppressing the lymphocyte activity which was suggested by Petrie et al., as Granulocytes possess the ability to inhibit Cytotoxic TLymphocytes (CTL) activity in vitro. This was seen also in Colorectal Cancer patients (CRC) patients as it was proven to decline in them than the healthy control in addition to gastric cancer patient [7]

On the contrary, the Absolute Neutrophilic Count (ANC) was significantly higher in the submucosal malignant and potentially malignant patients than that with the benign submucosal lesions and the healthy control. These results are in concordance with previous reports. There was significantly higher ANC in patient proven to have CRC than that of the healthy control. This can be explained as the neutrophils represent a major leucocyte subclass, which promote cancer cell proliferation, angiogenesis, and metastasis by the production of proangiogenic chemokines and growth factors such as Vascular Endothelial Growth Factor (VEGF).

A higher neutrophil count increases chemokine production such as (IL-1) (IL-6), and tumor necrosis factor, so enabling tumor progression. In addition, they-together with other phagocytic cells-induce DNA damage in proliferating cells, through their generation of reactive oxygen and nitrogen species 
that are produced normally in cases of infection or chronic inflammation and repeated tissue damage agent resulting in permanent genomic alterations such as point mutations, deletions, or rearrangements [8].

This explain the unsignificant statistical difference in total leukocytic count between malignant, benign submucosal lesions patients and healthy control as well which is further discussed by the subthreshold neoplastic states recognized by Peytoun Rous suggesting that chronic inflammation may lead a progressive neoplastic condition whether directly or indirectly, as it induce cell proliferation, recruit inflammatory cells, increase the production of reactive oxygen species leading to oxidative DNA damage which is reversible and reduce DNA repair resulting in DNA replication and proliferation of cells that have lost normal growth control [7].

Mariani et al., suggested a strong association between chronic inflammation and the development of CRC in individuals with inflammatory bowel diseases and between chronic helicobacter pylori infection and gastric MALT lymphoma which was proven in addition to other studies stating that nonsteroidal anti-inflammatory drugs reduce the systemic inflammation and the risk of CRC and have been investigated in various precancerous lesions and the hepatocellularcarcinoma associated with hepatitis [9-11].

This may be in the favor of the insignificant difference between the group of patient with benign submucosal lesion and the healthy control group together with the history of the longstanding upper GI dyspeptic symptoms together and epigastric pain for most of our patients.

So NLR increase in patients with malignant and potentially malignant submucosal lesions than that of the benign and the healthy control group.

In the current study, NLR was significantly higher in patient with malignant and potentially malignant submucosal lesions than those with benign ones and the healthy control. The best cut off value of NLR to discriminate between them was $>2.25$. This is in some similarity to a previous work in CRC in which cut off value of NLR $>2.15$ were the best to discriminate patient with malignant lesions from healthy control [7].

This concordance may be related to the entity of colorectal cancer as it includes various types of carcinoid or neuro-endocrine tumors, colorectal sarcoma which is a leiyomyosacroma in nature and GIST which can occur anywhere in GI tract, all of which are considered malignant or potentially malignant gastrointestinal submucosal lesions [7]

However on Zeren et al., studies that were conducted on a group of gastric adenocarcinoma where NLR were higher compared to the current study, but this may be explained by the high NLR accompanying gastric adenocarcinoma in relation with other submucosal lesions [12]

Many studies showed that platelets have been noted as promoters of metastases, due to their ability to coat tumor cells making them unrecognizable for the natural killer cells produced by the immune system. And the high levels of VEGF, PDGF, and PF4 may explain the new vessel development, as well as prevent bleeding from new vessels, leading to tumor cell promotion. Additionally, platelet VEGF was notably higher in cancer patients in comparison to healthy controls and that could be a possible explanation for the increased number of platelets $[13,14]$

Platelets count is proved to be higher also patients with malignant and potentially malignant submucosal lesions than patient with benign submucosal lesion and the healthy control where it has been found that thrombocytosis occurs as a result of stimulation of megakaryocytes by proinflammatory cytokines such as interleukin- 1 and interleukin- 6 which is triggered by the high neutrophilic count $[\mathbf{1 5 , 1 6}]$.

The aggregation of tumor cells and platelets increase the tumor cell viability. Thus, elevated PLR levels are related with carcinogenesis which was evident in association with low ALC in this study, a cut off value of PLR > 169 was higher than that in malice et al study on CRC patients in which the cut off value of PLR was 123.

The cut off value of the current study was in the same similarity with another study on gastric cancer patients which was $>160$ in discrimination of gastric cancer patient from healthy controls and proved to be decreasing after chemotherapy $[7,12]$

This was also reported in Kyu et al., study of which proved a higher PLR level in patients with solid ovarian masses than healthy control individuals [17].

\section{Conclusion:}

In conclusion, the main finding of our study was that the NLR and the PLR is significantly higher in patients with malignant and potentially malignant upper gastrointestinal tract submucosal 
lesion than the healthy control or benign submucosal lesions.

This is the first study to measure NLR and PLR in gastric submucosal lesions to be reported in this particular patient group.

The findings of this study mirrors similar observations in other solid organ malignancies, as ovarian cancer and malignant thyroid nodules when compared to patients with non-malignant disorders of the same organ, or to healthy controls. This study supports the combined diagnostic efficacy of NLR and PLR as a potential feasible and accessible combination added to upper gastrointestinal submucosal lesion detection, screening and predicting their behaviors in addition to other modalities, considering it is currently a part of routine blood work analysis.

\section{References}

1- POLKOWSKI M., LARGHI A., WEYNAND B., et al.: European Society of Gastrointestinal Endoscopy (ESGE). Learning, techniques, and complications of endoscopic ultrasound (EUS)-guided sampling in gastroenterology: European Society of Gastrointestinal Endoscopy (ESGE) Technical Guideline. Endoscopy, 2012.

2- SCHREIBER R.D., OLD L.J. and SMYTH M.J.: Cancer immuno editing: Integrating immunity's roles in cancer suppression and promotion. Science, 33 1: 1565-70, 2011.

3- RÖSCH T., KAPFER B., WILL U., BARONIUS W., STROBEL M., LORENZ R. and ULM K.: Accuracy of endoscopic ultrasonography in uppe gastrointestinal submucosal lesions: A prospective multicenter study. Scand. J. Gastroenterol., 37: 856-62, 2002.

4- OESTERHELWEG L., BRAND B., BINMOELLER K.F and SRIRAM P.V.: Impact of endoscopic ultrasound for evaluation of submucosal lesions in gastrointestinal tract. Dig. Liver Dis., 34: 290-7, 2002.

5- CHO J.W.: Current guidelines in the management of upper gastrointestinal subepithelial tumors. Clin. Endosc., 49: 235-40, 2016

6- VURAL V., SAYLAM B., COMCALı B., DUZGUN A.P., OZER M.V., et al.: D1 versus D2 dissection in gastric carcinoma: Evaluation of postoperative mortality and complications. Ulus Cerrahi Derg, 29: 1-6, 2013.

7- MILICA S., ALEKSANDRA P.C., SANJA S., MIRJANA S., IVAN D., IRENA R., et al.: Combined Diagnostic Efficacy of Neutrophil-to-Lymphocyte Ratio (NLR), Platelet-to-Lymphocyte Ratio (PLR), and Mean Platelet Volume (MPV) as Biomarkers of Systemic Inflammation in the Diagnosis of Colorectal Cancer, 2019.

8- MAEDA H. and AKAIKE T.: Nitric oxide and oxygen radicals in infection, inflammation, and cancer. Biochemistry, 63: 854-65, 1998.

9- MARIANI F., P. SENA and L. RONCUCCI: "Inflammatory pathways in the early steps of colorectal cancer development," World Journal of Gastroenterology, Vol. 20, No. 29, 2014

10-BURR N.E., M.A. HULL and V. SUBRAMANIAN: "Does aspirin or non-aspirin non-steroidal anti-inflammatory drug use prevent colorectal cancer in inflammatory bowel disease?," World Journal of Gastroenterology, Vol. 22, No. 13, pp. 3679-86, 2016.

11- ERNST P.B. and GOLD B.D.: The disease spectrum of Helicobacter pylori: The immunopathogenesis of gastroduodenal ulcer and gastric cancer. Annu. Rev. Microbiol., 54: 615-40, 2000.

12- ZEREN S., BAYHAN Z., OZBAY I., KAHRAMAN C., YAYLAK F., et al.: Mean Platelet Volume as a Biomarker for Thyroid Carcinoma. Int. Surg., Jul. 10, 2015.

13- NIESWANDT B., M. HAFNER, B. ECHTENACHER and D.N. MÄNNEL: "Lysis of tumor cells by natural killer cells in mice is impeded by platelets," Cancer Research, Vol. 59, No. 6, pp. 1295-300, 1999.

14- GOUBRAN H.A., J. STAKIW, M. RADOSEVIC and T. BURNOUF: "Platelets effects on tumor growth," Seminars in Oncology, Vol. 41, No. 3, pp. 359-69, 2014.

15-NASH G.F., TURNER L.F., SCULLY M.F. and KAKKAR A.K. : Platelets and cancer. Lancet Oncol., 3: 425-30, 2002.

16- ZHANG W., DANG S., HONG T. , TANG J., FAN J., et al.: A humanized single-chain antibody against beta 3 integrin inhibits pulmonary metastasis by preferentially fragmenting activated platelets in the tumor microenvironment. Blood, 120: 2889-98, 2012.

17- KYU EO, KI HYUNG KIM, EUN JOO PARK, HEUNG YEOL KIM, HONG-BAE KIM, et al.: Diagnostic accuracy of inflammatory markers for distinguishing malignant and benign ovarian masses, Journal of cancer, 320-4, 2019. 


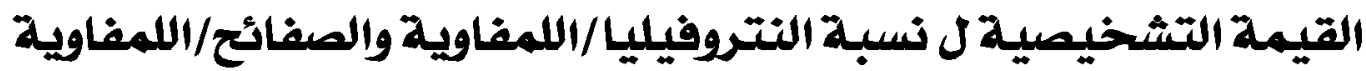

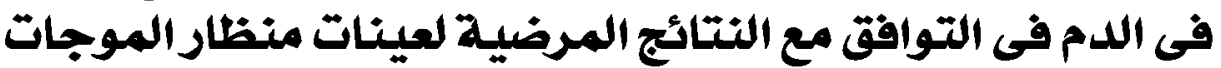

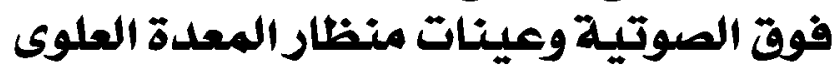

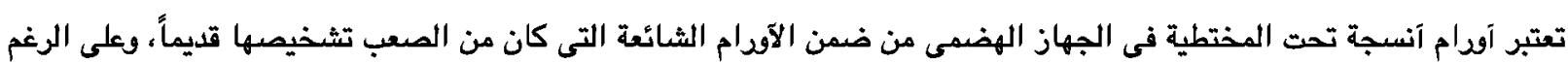

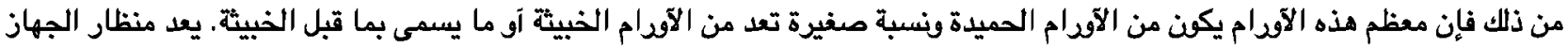

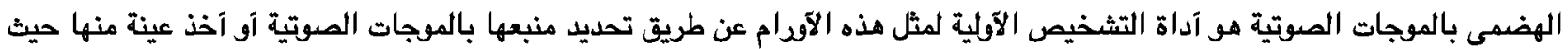

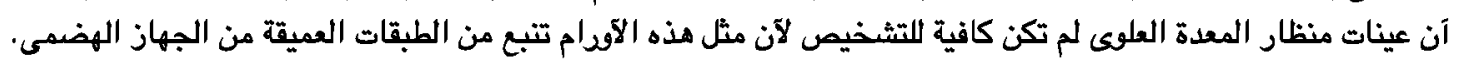

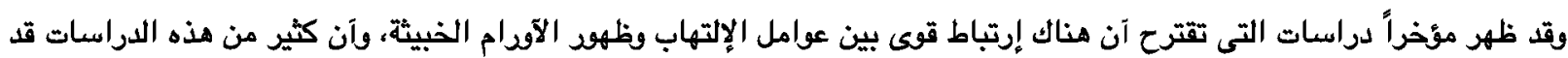

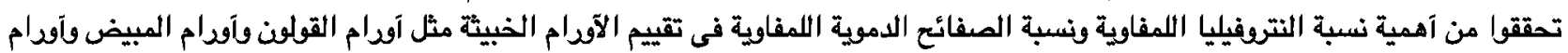

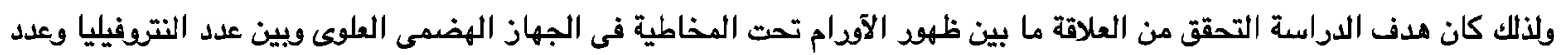

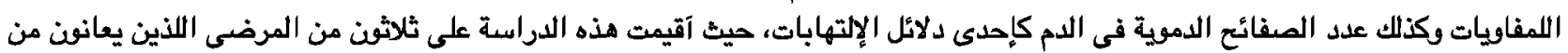

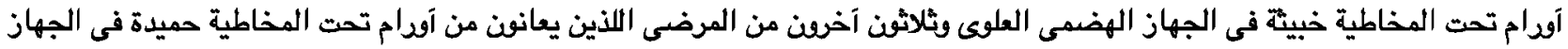

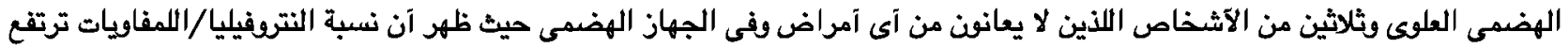

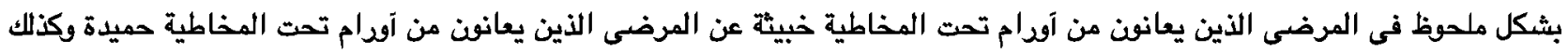

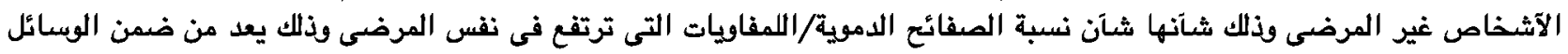

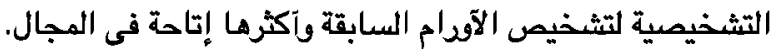

